क्ष

Off-Line Analysis Software for the Texas Test Rig

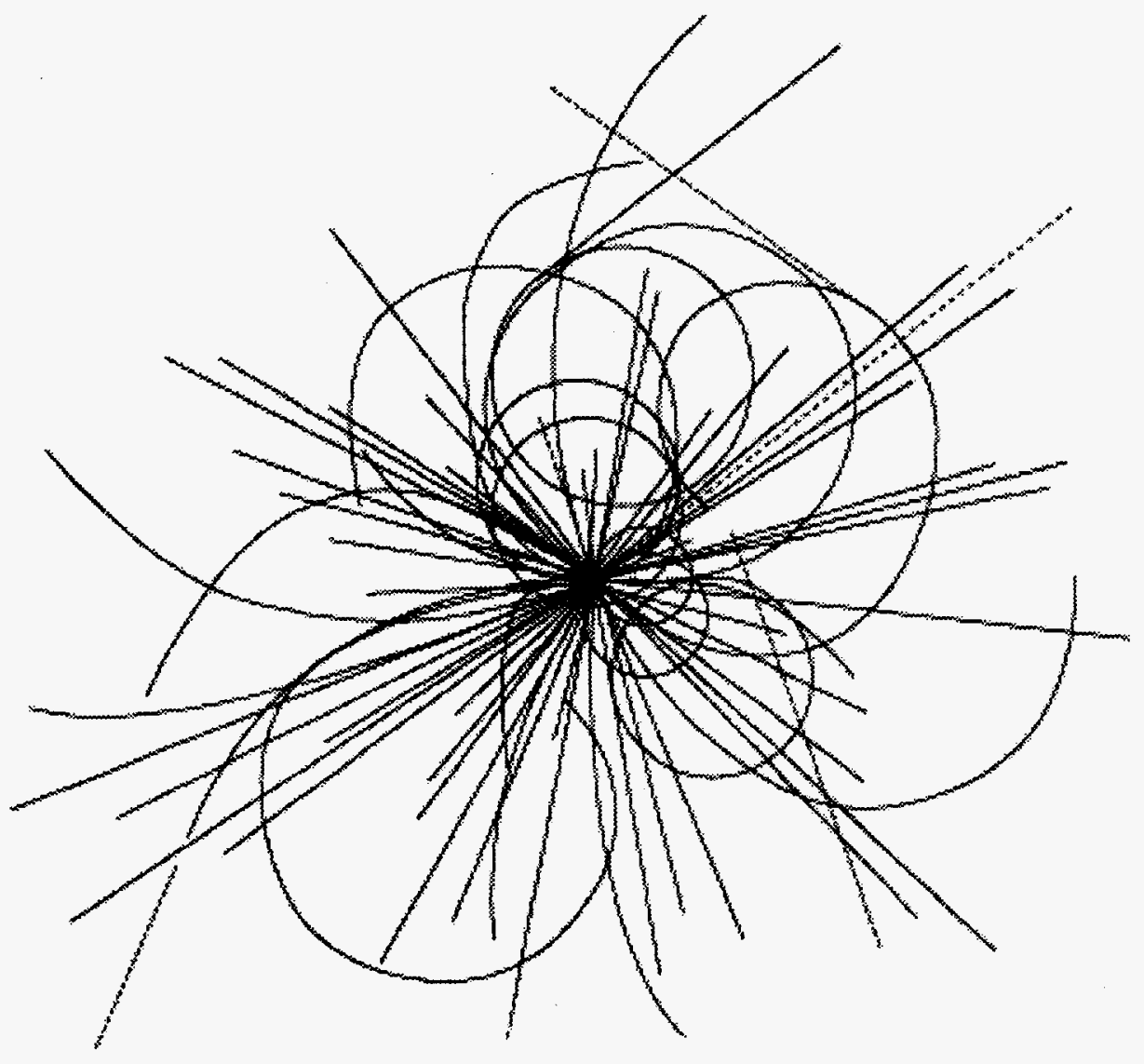

\section{Superconducting Super Collider Laboratory}

CONF-930537--120

SSCL-Preprint-479

May 1993

Distribution Category: 400

G. P. Yost
APPROVED FOR RELEASEOR:

PUBLICATION - OR. PATENTIGROUP BY...2.......DATEY/3/\%.5. 


\section{Disclaimer Notice}

This report was prepared as an account of work sponsored by an agency of the United States Govemment. Neither the United States Government or any agency thereot, nor any of their employees, makes any warranty, express or implied, or assumes any legal liability or responsibility for the accuracy, completeness, or usefulness of any information, apparatus, product, or process disclosed, or represents that its use would not infringe privately owned rights. Reference herein to any specific commercial product, process, or service by trade name, trademark, manufacturer, or atherwise, does not necessarily constitute or imply is endorsement, recommendation, or favoring by the United States Government or any agency thereot. The views and opinions of authors expressed herein do not necessarily state or reflect those of the United States Government or any agency thereot.

Superconducting Super Collider Laboratory is an equal opportunity employer. 


\section{DISCLAIMER}

Portions of this document may be illegible in electronic image products. Images are produced from the best available original document. 


\title{
Off-Line Analysis Software for the Texas Test Rig*
}

\author{
G. P. Yost \\ Superconducting Super Collider Laboratory ${ }^{\dagger}$ \\ 2550 Beckleymeade Ave. \\ Dallas, TX 75237
}

May 1993

"Presented at the Fifth Annual International Symposium on the Super Collider, May 6-8, 1993 San Francisco, CA.

${ }^{\dagger}$ Operated by the Universities Research Association, Inc., for the U.S. Department of Energy under Contract No. DE-AC35-89ER40486. 


\title{
OFF-LINE ANALYSIS SOFTWARE FOR THE TEXAS TEST RIG
}

\author{
G. P. Yost for the GEM Collaboration \\ Superconducting Super Collider Laboratory* \\ 2550 Beckleymeade Avenue \\ Dallas, TX 75237-3997
}

\begin{abstract}
Data analysis for the TTR requires integrating a large number of muon chamber technologies, each with different requirements, into a single analysis chain. Many of these technologies come with their own software, which have different conventions; these packages are grafted on. Data are stored on a tape robot with essential information stored in a database where it may be queried. Operation is done from special-purpose $\mathrm{X}^{\mathrm{TM}}$ windows designed to facilitate data selection and its subsequent analysis. Program development was done using the Hewlett-Packard Softbench ${ }^{\mathrm{TM}}$ product.
\end{abstract}

\section{INTRODUCTION}

The Texas Test Rig (TTR) is a 5 meter tall cosmic-ray telescope used to test and calibrate prototype muon chambers for the GEM Collaboration. Two planes of scintillator hodoscopes at the top and bottom provide a trigger. A one-meter thick steel filter which can be magnetized, located between the trigger planes and below the prototype chambers, restricts valid triggers to muons of energy above about $1.4 \mathrm{GeV} / \mathrm{c}^{2}$. Four planes of Iarocci chambers of $1 \mathrm{~cm}$ pitch provide straight-line determination above (two planes) and below (two planes) the steel. These are used, when the magnet is energized, to measure the bend angle and thereby enable the muon momentum to be estimated. In fact, the bend angle in both the "bend" and the "non-bend" planes (relative to the field) can be measured, allowing the amount of multiple scattering to be used as a second factor in the muon momentum measurement, as described in Vanyashin and Yost. 1

Further details on the TTR may be found in the paper by E. Cas Milner, submitted to this conference.

The data for the TTR are acquired as described by Luis Villasenor in this conference. The data are then split into two streams. The first stream contains the complete data before zero-suppression; this is stored on an 8-mm tape. The second stream contains that portion of

\footnotetext{
* Operated by the Universities Research Association, Inc., for the U. S. Department of Energy under Contract No. DE-AC35-89ER40486.

${ }^{1}$ A. Vanyashin and G. Yost, "Monte Carlo Studies of the Texas Test Rig Performance," GEM TN-92-99 (1992).
} 
the data surviving zero suppression; this is stored on a disk. There is also an event display which is accessible on-line.

The off-line software is responsible for moving this zero-suppressed data to a tape robot for permanent storage, for entering indicative information regarding this data into a database, and for the analysis of this data. We proceed to describe the philosophy and practice of this software.

\section{RUNNING THE OFF-LINE SOFTWARE}

The TTR data flow is shown in Figure 1. The data come from the on-line system to reside temporarily on a disk. At regular intervals (e.g., every 15 minutes) the disk is queried for the presence of new data. The presence of new data is signified by a file inserted into a particular directory with a particular name structure. These files are not written until a run is complete. The query is initiated by a SYBASE" database server running on the "Physics/ Detector Simulation Facility" (PDSF) at the SSC Laboratory.

When a new data file is present, it is retrieved over the network and stored on a tape robot. Certain indicative records, always present at the beginning and the end of the file, are scanned. These records contain information on the technologies which are taking data, the number of triggers, the temperature and humidity at the start and the end of the run, and other information. This indicative information is stored in the database along with a pointer to the associated data on the robot.

Once the data file has been stored, the filename on the temporary disk is changed. If the network should go down before the transfer is complete, this last step is not accomplished and the data file will be retrieved anew when the network returns. In this case, any portion of the file which may have been accessed prior to the network problem is over-written.

Data may now be analyzed using a package which currently runs on an HP 9000/730 workstation. A pop-up window presents three choices: query the database, run the analysis program, or run the event display.

In inverse order, the event display is identical with the on-line version and will not be discussed further. To run the analysis program a new pop-up window appears. In this window one must specify files to be processed. These may be located on the PDSF robot or on a local disk. The window calls for a file containing a list of files, both for the robot files and for the local files. Each such list of files may be constructed and/or edited from the window. The robot has a typical access time of about five minutes, so one is well-advised to transfer data files to a disk if frequent access is desired. The off-line program will read any number of files, beginning with the tape robot and proceeding to the disk when desired files off the robot are completed.

The off-line program also requires two files containing survey data and calibration data. Each file is in binary format and can be changed only by running a special program. This is to ensure that quick changes don't run the risk of creating a file which can not be read. The names of these files are entered in the off-line window; defaults are provided.

The off-line program has one additional running feature. The user may substitute his or her own special subroutines in place of standard routines. The user must place all such routines in a special archive file which is specified in the window. The program is then loaded dynamically, so that routines are loaded when called. The loader looks in this archive file first, then in the standard files, when loading a routine which has been called. Routines which happen not to be called are not loaded, and need not be present.

This subroutine-substitution feature is essential for a program which is intended to be used by a variety of people from different institutions. Each user will want to install their own code to analyze the data from their own chambers. 
The final choice in the primary window is to access the database. This offers in its turn a set of windows with selections which are automatically entered into a database query. One may, for example, select runs featuring a certain set of chambers running with certain gases. One may select temperature and/or humidity ranges, magnet current in a range, and a range of run numbers. One may select a range of trigger counts, e.g., more than 1000 to ensure processing only of runs with adequate statistics.

Following the selections, one may press "fetch." The database typically returns the results in a few seconds. A window displays the filenames on the robot and the associated run numbers and list of the technologies active for that run.

Clicking on a particular run causes the complete set of database information for that run to be displayed in another window. This information may be printed. Finally, the results of the selections are passed to the off-line program window described above.

\section{STRUCTURE OF THE ANALYSIS SOFTWARE}

The analysis software is structured to provide "hooks" for user routines which may be substituted for dummy routines provided with the standard package. It also accomplishes a number of other tasks. It will read and unpack the data. It will read the survey file and store the surveyed locations of the chambers for later use (e.g., so that a given track could be followed from one set of chambers to another). It will read the calibration file and apply the calibration constants to, e.g., convert ADC counts to induced charge.

The program then proceeds to process the track. It makes an estimate of the location in the scintillators which was struck. It attempts to correlate this with Iarocci information and construct a track from the combined information both above and below the steel. If, as is sometimes the case, some of the Iarocci data are missing, it uses what information it does have to make an estimate of the trajectory. If a complete trajectory (both above and below the magnet) in either the bend or the non-bend plane, or both, is available, it is then used to estimate the momentum.

When this is done, the program calls standard routines (or their user-supplied replacements) to reconstruct the track in the prototype chambers and calculate a $\chi^{2}$ for the fit.

This will be done for all tracks in the data files requested. If desired, selected summary data for each track may be written to disk for later input to a global fit. In such a fit, quantities such as survey locations or calibration constants may be varied to find optimized values for the collected set of tracks. This requires rerunning the entire set of tracks for each iteration, of course, and can therefore be very time-consuming.

\section{SUMMARY}

An off-line data storage, retrieval, and analysis package with novel features has been constructed for data collected by the Texas Test Rig. This package has successfully stored all the TTR data on a tape robot with indicative data stored in a SYBASE ${ }^{\mathrm{TM}}$ database. The program queries this database, retrieves desired data, and processes it in an analysis package. 

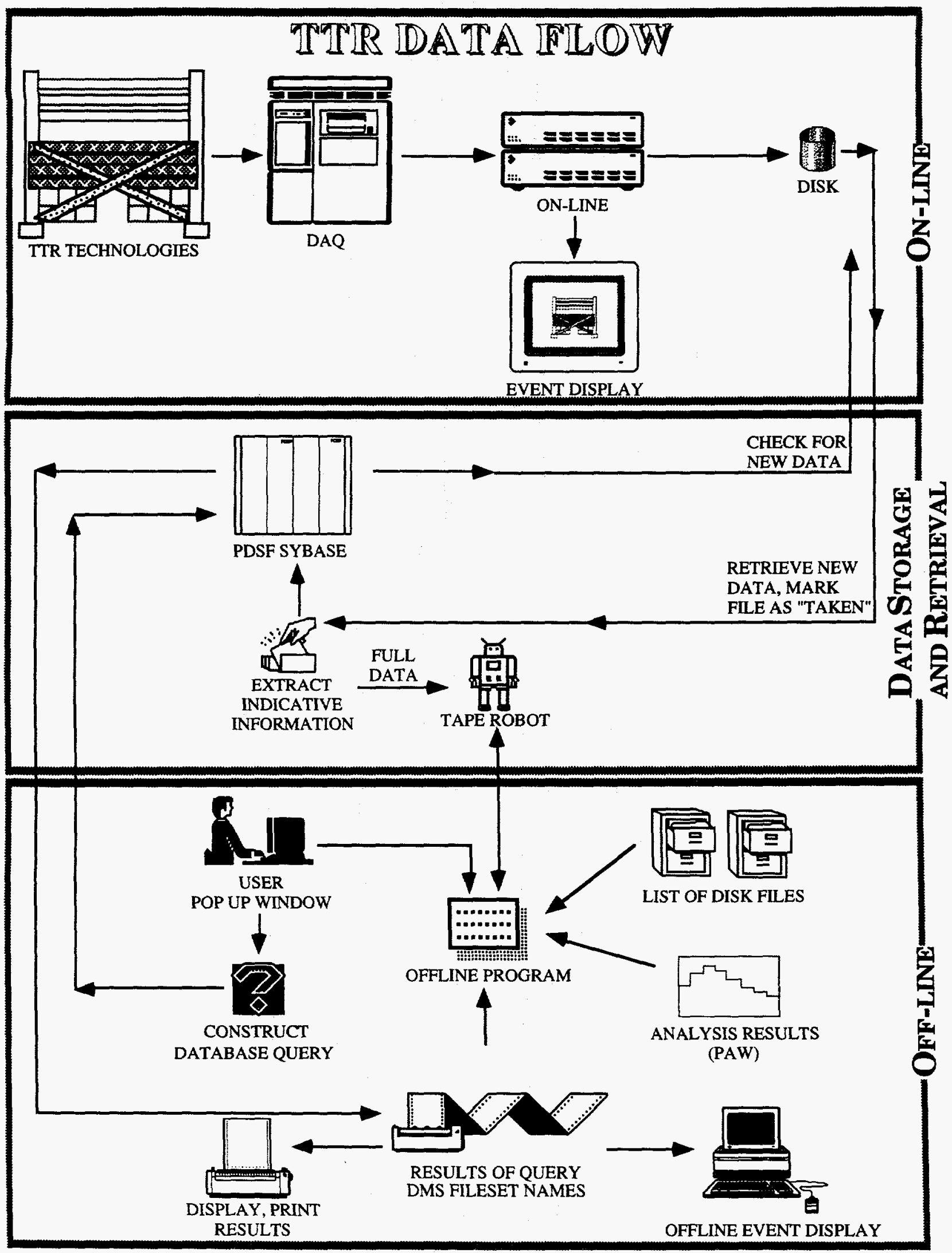

Figure 1. Texas Test Rig (TTR) Data Flow. 